\title{
Das Kriegsgeschehen 2013
}

\author{
Wolfgang Schreiber*
}

\begin{abstract}
In 2013 the Hamburg Working Group for Research on the Causes of War (Arbeitsgemeinschaft Kriegsursachenforschung, AKUF) registered 22 wars and 8 armed conflicts. This is the lowest number of wars in the AKUF data base since 1962 . The article provides some data on the distribution of these conflicts by region and war types. Furthermore, each of these conflicts and especially major changes during the year 2013 are described in brief.
\end{abstract}

Keywords: Krieg, bewaffneter Konflikt

War, armed conflict

I m Jahr 2013 registrierte die Hamburger Arbeitsgemeinschaft Kriegsursachenforschung (AKUF) 22 Kriege und acht bewaffnete Konflikte. Damit ist die Gesamtzahl der

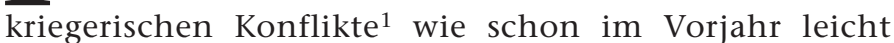
zurückgegangen. Sie verringerte sich von 34 auf $30 .^{2}$ Als beendet sind insgesamt sieben kriegerische Konflikte zu betrachten. Umgekehrt eskalierten 2013 drei bewaffnete Konflikte neu.

Der Höhepunkt des Kriegsgeschehens seit 1945 wurde nach den Daten der AKUF 1992 mit damals 55 Kriegen erreicht. Diese Zahl hat sich bis 2013 um 60 Prozent auf 22 verringert. Eine geringere Anzahl von Kriegen gab es zuletzt 1962. Nicht ganz so deutlich sank die Gesamtzahl der Kriege und bewaffneten Konflikte, die 1993 erstmals mit 63 erfasst wurde und auf 30 im Jahr 2013 zurückging. Dieser Rückgang ist trotz einiger Schwankungen vergleichsweise kontinuierlich verlaufen (siehe Grafik). Allerdings lässt sich aus diesem Trend der letzten 20 Jahre nicht einfach eine Prognose für die Zukunft ableiten: Einerseits lässt sich das Entstehen neuer kriegerischer Konflikte kaum verlässlich voraussagen. Andererseits ist ungewiss, ob ein auch 2013 in mehreren Fällen zu beobachtendes Nachlassen der Kampfhandlungen oder auch Verhandlungen zwischen den Konfliktparteien zur Beendigung dieser kriegerischen Konflikte führen werden.

\section{Kriege}

Von den 22 im Jahr 2013 geführten Kriegen wurden allein neun in Afrika ausgetragen. In Asien waren es sieben und in der Region des Vorderen und Mittleren Orients fünf. In Südamerika war noch ein Krieg zu verzeichnen. Soweit die regionale Verteilung. Schaut man auf die Kriegstypen, so wurden 21 der 22 Kriege 2013 als innerstaatliche Kriege klassifiziert, davon 13 als Antiregime- und acht als Sezessions- oder Autonomiekriege. Die einzige Ausnahme bildete der Krieg in Afghanistan, wo neben der afghanischen Armee auch die USA weiterhin einen originären Kriegsakteur darstellten. Allerdings ist darüber hinaus festzustellen, dass in sieben der 21 und damit immerhin in einem Drittel der innerstaatlichen Kriege 2013 mindestens ein fremder Staat mit Truppen interveniert hat.

* Diplom-Mathematiker. Lehrbeauftragter und Leiter der Arbeitsgemeinschaft Kriegsursachenforschung (AKUF) am Institut für Politikwissenschaft der Universität Hamburg.

1 Der Begriff „kriegerische Konflikte“ dient in diesem Text als Oberbegriff für Kriege und bewaffnete Konflikte.

2 Zu den Daten für 2012 vgl. Schreiber, Wolfgang: Kriege und bewaffnete Konflikte 2012. Ein erster Überblick, AKUF-Analysen Nr. 11, Hamburg 2012.
Gegenüber dem Vorjahr hat sich die Zahl der Kriege um zwei verringert. Insgesamt wurden drei Kriege beendet und einer neu begonnen. Von diesen vier Veränderungen wiederum lag bei dreien lediglich ein Wechsel der definitionsgemäßen Einstufung vom Krieg zum bewaffneten Konflikt bzw. umgekehrt vor. Lediglich ein Krieg kann als so weit beendet betrachtet werden, dass er auch nicht mehr als bewaffneter Konflikt fortgeführt wird.

Dabei handelt es sich um den Konflikt zwischen Israel und verschiedenen palästinensischen Gruppen. Dieser eskalierte zuletzt nach der Tötung des Hamas-Militärchefs Ahmed alJaabari bei einer israelischen Militäroperation im November 2012. Danach blieb Gewalt nicht aus. So gab es auch 2013 noch zahlreiche Tote bei Auseinandersetzungen zwischen israelischen Sicherheitskräften und Palästinensern, insbesondere bei Razzien in der Westbank. Auch wurden israelische Soldaten Opfer von gezielten Attentaten. Die Zahl der aus dem Gaza-Streifen abgefeuerten Raketen erreichte 2013 in keinem Monat mehr als ein Dutzend, während im November 2012 noch über 1.500 abgefeuert worden waren. Insgesamt kam es 2013 jedoch kaum noch zu Kampfhandlungen im engeren Sinne.

In der Türkei führten die Gespräche zwischen der Regierung und der Partiya Karkeren Kurdistan (PKK) zwar nicht zu einer Einstellung, jedoch zu einem deutlichen Rückgang der Kampfhandlungen. Daher konnte nicht mehr von einem Krieg, aber aufgrund mehrerer Zusammenstöße doch noch von einem bewaffneten Konflikt gesprochen werden. Insbesondere im letzten Quartal des Jahres kam der Friedensprozess ins Stocken, da die Zugeständnisse zugunsten des kurdischen Teils der Bevölkerung bis dahin geringer ausfielen als erhofft.

In Äthiopien schwächte sich der Krieg mit den Rebellen der Ogaden National Liberation Front (ONLF) ebenfalls zum bewaffneten Konflikt ab. Allerdings wurde hier schon in der Vergangenheit jede Einstufung des Konflikts aufgrund einer massiven Informationsblockade seitens der Regierung erschwert. Angaben über Kampfhandlungen stammten fast ausschließlich von den beiden Kriegsparteien. 2013 berichtete fast ausschließlich die ONLF noch über Kämpfe.

Der einzige neue Krieg des Jahres 2013 wurde im Südsudan geführt. Hier hatten sporadische Kämpfe bereits seit 2010 stattgefunden. Sieht man von Übergriffen von Milizen verschiedener ethnischer Gruppen ab, die mehrere Hundert Menschen das Leben kosteten, waren an dem Konflikt vor allem ranghohe Mitglieder der Regierungspartei Sudan People's Liberation Movement (SPLM) beteiligt, die für sich in dem neuen unabhängigen Staat Südsudan eine 
Abbildung 1: Kriege und bewaffneten Konflikte

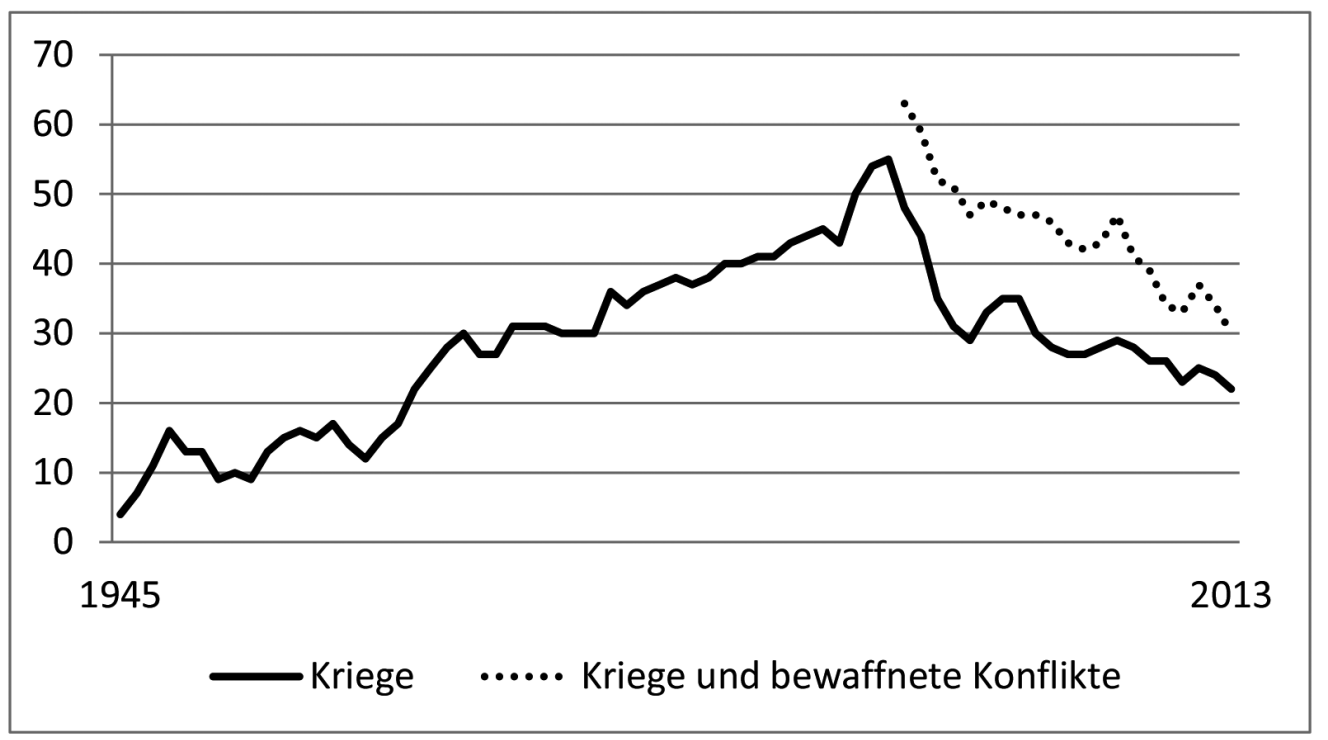

Die größte Aufmerksamkeit erfuhr der Krieg in Somalia 2013 durch den im September verübten Angriff auf ein Einkaufszentrum in der kenianischen Hauptstadt Nairobi, bei dem Dutzende Menschen getötet und Hunderte verletzt wurden. Dieses war eine wenn auch späte Vergeltung dafür, dass die islamistischen Rebellen der AlShabaab 2012 mit kenianischer Hilfe aus dem Westen Somalias und insbesondere aus der wichtigen Hafenstadt Kismayo zurückgedrängt worden waren. Kontinuierliche Kampfhandlungen fanden 2013 in Somalia vor allem in den Regionen Lower Shebelle und Bakol statt. Aber auch in der Hauptstadt Mogadischu blieb AlShabaab mit diversen Anschlägen

größere Rolle angestrebt hatten. Fast das ganze Jahr 2013 fanden Kämpfe zwischen Regierungstruppen und Rebellen unter dem ehemaligen General David Yau Yau im Bundesstaat Jonglei statt. Endgültig eskalierte der Konflikt am 17. Dezember, nachdem Präsident Salva Kiir den im Juli von ihm entlassenen Vizepräsidenten Riek Machar eines Putschversuchs beschuldigt hatte.

\subsection{Kriege in Afrika}

In Afrika wurde im Vorjahr ein Krieg, nämlich der in Äthiopien, nur noch als bewaffneter Konflikt eingestuft. Umgekehrt eskalierte der bewaffnete Konflikt in Südsudan zum Krieg (s.o.). Daher blieb die Gesamtzahl der Kriege wie im Vorjahr bei neun. Von diesen wurden alle als Antiregimekriege ausgetragen. In zwei Dritteln, d.h. in sechs der neun Kriege, war zudem 2013 eine militärische Intervention zu beobachten.

Der Sudan blieb 2013 Schauplatz von zwei Kriegen. Die Kämpfe in der Region Darfur, vor allem mit Rebellen der Justice and Equality Movement (JEM), dauerten das ganze Jahr über an. Intensiver waren die Kampfhandlungen zwischen Regierungstruppen und der Sudan People's Liberation Army - North (SPLA-N), dem nördlichen Ableger der früheren südsudanesischen Rebellengruppe SPLA, die vor allem in den Provinzen Süd-Kordofan und Blue Nile ausgetragen wurden. Beide Kriege im Sudan hatten in der Vergangenheit eine sezessionistische Komponente bzw. wurden explizit für eine größere Autonomie geführt. Mittlerweile beanspruchen die beiden stärksten Rebellengruppen aber, vor allem für einen Sturz der Regierung zu kämpfen. Zu diesem Zweck schlossen sie sich zur Sudan Revolutionary Front (SRF) zusammen. Allerdings operierten sie zumindest teilweise auch noch separat, sodass nicht völlig klar ist, wie unabhängig die beiden Gruppen noch sind oder ob nicht die ursprünglich zwei Kriege mittlerweile nur noch einen darstellen. In allen zwischen Regierung und Rebellen umkämpften Provinzen fanden 2013 darüber hinaus Kampfhandlungen zwischen oder Übergriffe durch Milizen ethnischer Gruppen statt, die für den größten Teil der Todesopfer im Sudan verantwortlich waren. präsent. Obwohl es zu Spaltungen innerhalb Al-Shabaab kam, bei denen über 100 Rebellen getötet wurden, blieb diese offensichtlich eine erhebliche Bedrohung. Der UN-Sicherheitsrat stimmte im November einer Aufstockung der afrikanischen Eingreiftruppe AMISOM um 4.000 Personen zu.

Der von Uganda gegen die Lord's Resistance Army (LRA) unter ihrem Anführer Joseph Kony geführte Krieg, fand seit seinem erneuten Beginn 2008 nie auf ugandischen Territorium statt. Austragungsorte waren die Demokratische Republik Kongo, der Südsudan und 2013 vor allem die Zentralafrikanische Republik, wo ugandische Truppen mit Unterstützung der jeweiligen Regierungen gegen die LRA vorgingen. Da alle drei Länder auch von eigenen Kriegen betroffen waren, welche die Berichte dominierten, ist das Ausmaß der Kampfhandlungen mit der LRA im Jahr 2013 schwierig zu beurteilen.

Im Osten der Demokratischen Republik Kongo wurde 2013 zwischen der Regierung und der Rebellengruppe M23 sowohl verhandelt als auch gekämpft. Waren die Rebellen im November 2012 militärisch mit der Eroberung der Provinzhauptstadt Goma noch sehr erfolgreich gewesen, so räumten sie diese allerdings nach internationalem Druck und Versprechungen der Regierung zu ernsthaften Verhandlungen schon Anfang Dezember wieder. Dieser Druck erhöhte sich 2013 erheblich, als die UN zusätzlich zur bereits bestehenden MONUSCO-Mission (Mission de l'Organisation des Nations Unies en République démocratique du Congo) eine Interventionsbrigade mit offensivem Auftrag beschlossen. Nachdem diese, hauptsächlich aus tansanischen und südafrikanischen Soldaten bestehende 3.000 Mann starke Truppe zum Einsatz gekommen war, blieb der M23 Anfang November keine andere Möglichkeit mehr, als um einen Waffenstillstand zu bitten. Damit dürfte der Krieg im Kongo allerdings kaum beendet sein. Seit 2005 haben sich mehrere Rebellengruppen und Milizen nacheinander im Osten Kongos als ernsthafte Herausforderung für die Regierung in Kinshasa dargestellt. Ungelöst ist vor allem noch das Problem mit den Forces Démocratiques de Libération du Rwanda (FDLR), die aus Milizen hervorgegangen sind, die 
für den Völkermord in Ruanda von 1994 verantwortlich waren. Darüber hinaus sind im Grenzgebiet zu Uganda die von dort stammenden Allied Democratic Forces (ADF) aktiv, die zuletzt mehrfach als nächstes Ziel der Interventionsbrigade genannt wurden.

Der Krieg in der Zentralafrikanischen Republik schien bereits mehrfach beendet zu sein. Im August 2012 konnte die damalige Regierung mit der letzten verbliebenen Rebellengruppe einen Friedensvertrag schließen, der jedoch nicht von allen Rebellen anerkannt wurde. Schon im November wurden die Kampfhandlungen wieder aufgenommen, an denen sich auch Rebellen beteiligten, die bereits zuvor Vereinbarungen mit der Regierung unterzeichnet hatten. Diese Rebellen, die unter dem Namen Seleka agieren, unterzeichneten im Januar einen Waffenstillstand und Anfang Februar wurde eine Übergangsregierung unter Einschluss des bisherigen Präsidenten Bozizé und der Seleka gebildet. Diese hatte allerdings keine zwei Monate Bestand: Am 24. März eroberten die Seleka-Rebellen die Hauptstadt Bangui und übernahmen die Regierung. Vor allem in der Hauptstadt entwickelten sich in der Folge Kämpfe zwischen den ehemaligen Seleka-Rebellen und in Milizen organisierten Anhängern des ins Exil geflohenen Präsidenten. Diese erreichten ein solches Ausmaß, dass Anfang Dezember das bereits vorhandene französische Kontingent zur Sicherung des Flughafens mit Zustimmung des UN-Sicherheitsrates vergrößert wurde. Auch die bereits in der Zentralafrikanischen Republik präsente Mission der AU wurde um zusätzliche Soldaten erweitert.

Im Norden Nigerias forderten die Kampfhandlungen zwischen der islamistischen Boko Haram und nigerianischen Sicherheitskräften 2013 weit über 1.000 Todesopfer. Außer Polizei- und Militäreinrichtungen gehörten insbesondere auch Schulen zu den Zielen von Angriffen von Boko Haram. Aber auch die nigerianische Regierung wurde für ihr Vorgehen gegen die Rebellen, bei denen es immer wieder zu zivilen Opfern kam, im In- und Ausland kritisiert.

In Mali wurde der 2012 begonnene Krieg zunächst von separatistischen Rebellen für die Unabhängigkeit Nordmalis geführt. Auch aufgrund der Unterstützung islamistischer Gruppen sowie eines Putsches in der Hauptstadt gelang es den Rebellen rasch, den Norden des Landes unter ihre Kontrolle zu bringen. Allerdings zerbrach das Bündnis zwischen den nach Unabhängigkeit strebenden Tuareg-Rebellen und den islamistischen Gruppen bereits nach wenigen Monaten. Dabei hatten die Tuareg-Rebellen schnell das Nachsehen und wurden von den Islamisten aus allen wichtigen Gebieten des Nordens verdrängt. Die Einsatzbereitschaft einer von den UN prinzipiell autorisierten und von der westafrikanischen Regionalorganisation ECOWAS geplanten Interventionstruppe wurde frühestens in der zweiten Jahreshälfte 2013 erwartet. Daher kam die französische Intervention am 11. Januar 2013 etwas überraschend. Innerhalb eines Monats wurden die Rebellen aus den größeren Städten im Norden weitgehend vertrieben. Im Umland fanden danach noch einige Militäroperationen statt und die islamistischen Rebellen verlegten sich von der Gebietskontrolle auf Anschläge. Die Rückkehr zur Normalität in Mali sollte durch Präsidentschaftswahlen im Juli und August dokumentiert werden. Allerdings blieb die Sicherheitslage in Nordmali angespannt.
Tabelle 1: Kriege 2013

\begin{tabular}{|c|c|c|}
\hline & Kriegstyp & Beginn \\
\hline \multicolumn{3}{|l|}{ Afrika } \\
\hline Kongo-Kinshasa (Ostkongo) & A-1 & 2005 \\
\hline Mali & A-1 & 2012 \\
\hline Nigeria (Boko Haram) & A-2 & 2011 \\
\hline Somalia & A-1 & 1988 \\
\hline Sudan (Darfur) & A-2 & 2003 \\
\hline Sudan (Südkordofan) & $\mathrm{A}-2$ & 2011 \\
\hline Südsudan & A-1 & 2013 \\
\hline Uganda (LRA) & A-1 & 2009 \\
\hline Zentralafrikanische Republik & A-1 & 2006 \\
\hline \multicolumn{3}{|l|}{ Vorderer u. Mittlerer Orient } \\
\hline Afghanistan & AE-1 & 1978 \\
\hline Irak & A-2 & 1998 \\
\hline Jemen (Al-Qaida) & A-1 & 2010 \\
\hline Russland (Nordkaukasus) & $\mathrm{B}-2$ & 1999 \\
\hline Syrien & A-2 & 2011 \\
\hline \multicolumn{3}{|l|}{ Asien } \\
\hline Indien (Kaschmir) & B-2 & 1990 \\
\hline Indien (Naxaliten) & A-2 & 1997 \\
\hline Myanmar & B-2 & 2003 \\
\hline Pakistan (Taliban) & $\mathrm{AB}-1$ & 2007 \\
\hline Philippinen (Mindanao) & B-2 & 1970 \\
\hline Philippinen (NPA) & $\mathrm{A}-2$ & 1970 \\
\hline Thailand (Südthailand) & $\mathrm{B}-2$ & 2004 \\
\hline \multicolumn{3}{|l|}{ Südamerika } \\
\hline Kolumbien & A-2 & 1964 \\
\hline
\end{tabular}

\subsection{Kriege in Asien}

In Asien waren 2013 sieben Kriege zu verzeichnen. Die Zahl blieb gegenüber dem Vorjahr unverändert, da weder Kriege beendet noch neue begonnen wurden. Alle sieben Kriege waren innerstaatlich, wovon vier für eine Unabhängigkeit oder größere Autonomie geführt wurden und zwei als Antiregimekriege einzustufen sind. Der Krieg in Pakistan ist hinsichtlich der Ziele der Rebellen nicht eindeutig einzuordnen. Dies ist auch der einzige Krieg in Asien, der mit den Drohnenangriffen der USA eine direkte Fremdbeteiligung aufwies.

In Indien wurden unverändert zwei Kriege ausgetragen. Am bedeutendsten hinsichtlich des Ausmaßes der Kampfhandlungen und der betroffenen Gebiete erwiesen sich die Auseinandersetzungen mit den als Naxaliten bezeichneten maoistischen Rebellen. Diese waren 2013 in fünf der 28 Unionsstaaten Indiens in größerem Maßstab aktiv, vor allem in Chhattisgarh und Jharkhand. Dabei wurden 2013 mehr als 400 Menschen getötet. Der nicht zuletzt wegen seiner Verbindung zum indisch-pakistanischen Konflikt bekanntere unter den Kriegen in Indien ist der Kaschmirkrieg. Nachdem sich dieser Krieg - ähnlich wie der mit den Naxaliten über mehrere Jahre abgeschwächt hatte, gab es 2013 erstmals 
Tabelle 2: Bewaffnete Konflikte 2013

\begin{tabular}{|l|c|}
\hline & Beginn \\
\hline Ägypten & 2013 \\
\hline Algerien & 1992 \\
\hline Äthiopien (Ogaden) & 2006 \\
\hline Indien (Assam) & 1990 \\
\hline Libyen & 2011 \\
\hline Mosambik & 2013 \\
\hline Tunesien & 2013 \\
\hline Türkei (Kurden) & 2004 \\
\hline
\end{tabular}

wieder einen leichten Anstieg bei den Opferzahlen: Etwa 180 Menschen kamen bei Auseinandersetzungen zwischen indischen Sicherheitskräften und Rebellen ums Leben.

In Pakistan dauerten die intensiven Kämpfe zwischen TalibanGruppen und der Armee sowie Anschläge der Rebellen an. Über 5.000 Menschen verloren hier ihr Leben. Zwei Drittel davon wurden bei Kampfhandlungen in den nördlichen Provinzen des Landes getötet. Dort wurden auch durchschnittlich zwei Drohnenangriffe der USA pro Monat beobachtet. Dies stellt eine Halbierung gegenüber dem Vorjahr dar und ist die niedrigste Zahl seit fünf Jahren. In der Provinz Sindh waren vor allem Schiiten das Ziel von Anschlägen der Taliban und ihnen nahestehender Gruppen.

Zwei Kriege geringeren Ausmaßes waren auch 2013 noch auf den Philippinen zu verzeichnen. Diese beiden Kriege begannen jeweils bereits 1970. Im Süden des Landes kämpfen Rebellen der philippinischen Muslime für mehr Autonomie der Region Mindanao. Die Moro Islamic Liberation Front (MILF) als größte Rebellengruppe führte das ganze Jahr über Friedensverhandlungen mit der Regierung und war kaum noch an Kampfhandlungen beteiligt. Kompliziert wird eine Lösung dieses Konflikts allerdings durch das Entstehen neuer Gruppen. Darüber hinaus ist auch die Moro National Liberation Front (MNLF), die bereits vor Jahren mit der Regierung Frieden geschlossen hatte, wieder aktiv geworden. Auf deren Konto geht auch der größte Angriff im Jahr 2013. Anfang September starben dabei in Zamboanga etwa 200 Menschen. Im zweiten Krieg ist die New People's Army (NPA) als militärischer Arm der Communist Party of the Philippines (CPP) aktiv. Hier ereigneten sich das ganze Jahr über kleinere Kämpfe mit Polizei und Militär.

Die bewaffneten Auseinandersetzungen in Myanmar, dem früheren Birma, begannen bereits 1948. Der Konflikt eskalierte nach einer kurzen Phase des Rückgangs der Kampfhandlungen 2003 erneut zum Krieg. Diverse Rebellenbewegungen ethnischer Gruppen kämpfen dabei für die Unabhängigkeit. Die Kachin Independence Organization (KIO) blieb die einzige Rebellengruppe, die 2013 regelmäßig in bewaffnete Auseinandersetzungen mit Regierungstruppen verwickelt war: Nachdem alle anderen Rebellengruppen bereits in den Vorjahren Waffenstillstandsabkommen mit der Regierung geschlossen hatten, wurden ab Ende Mai auch Gespräche mit der KIO geführt.

In Thailand streben muslimische Rebellen die Unabhängigkeit des Südens des Landes an. In den vorangegangenen Jahren wurde vor allem ziviles staatliches Personal von den Rebellen angegriffen. 2013 waren vermehrt Angriffe und Anschläge auf Angehörige von Polizei und Militär zu verzeichnen. Die Regierung verlängerte 2013 mehrfach den Ausnahmezustand in den südlichen Provinzen. Zugleich begannen im Februar von Malaysia vermittelte Gespräche mit der Barisan Revolusi Nasional (BRN), die aber bislang noch zu keinem Erfolg führten.

\subsection{Kriege im Vorderen und Mittleren Orient}

Im Vorderen und Mittleren Orient (VMO) wurden 2013 fünf Kriege geführt. Dies bedeutet gegenüber dem Vorjahr zwei Kriege weniger. Beendet wurde der zwischen Israel und palästinensischen Gruppen (s.o.). Ebenfalls beendet wurde der Krieg in der Türkei mit der PKK, wobei dieser allerdings noch als bewaffneter Konflikt fortgesetzt wurde (s.o.). Von den vier innerstaatlichen Kriegen im VMO sind drei als Antiregimekriege einzustufen und einer wird um die Unabhängigkeit geführt. Als nicht rein innerstaatlich war 2013 aufgrund der Eigeninteressen der USA der Krieg in Afghanistan einzustufen. Eine Fremdbeteiligung aufgrund von Drohnenangriffen durch die USA wies darüber hinaus der Krieg im Jemen auf.

Von allen Kriegen im VMO erhielt der in Syrien 2013 nicht zuletzt aufgrund der hohen Opferzahlen mit über 100.000 Toten seit seinem Beginn die größte Aufmerksamkeit. Seit März 2011 fanden von der Regierung gewaltsam unterdrückte Massenproteste statt. Spätestens mit Gründung der Free Syrian Army (FSA) im Herbst 2011 eskalierten diese zum Krieg. Das bedeutendste Einzelereignis des Krieges in Syrien war 2013 ohne Zweifel ein Giftgasangriff in der Nähe von Damaskus am 21. August, bei dem über 1.300 Menschen getötet wurden. Als dessen Folge wurde mit der Regierung ein internationales Abkommen zur Vernichtung aller syrischen Chemiewaffen geschlossen. Die Seite der Rebellen zeichnete sich durch eine große Fraktionierung in zahlreiche Gruppen aus.

Der Krieg in Afghanistan stand 2013 im Zeichen von für 2014 angekündigten Truppenabzügen der USA und der International Security Assistance Force (ISAF). Im Juni übernahmen die afghanischen Sicherheitskräfte offiziell die Führung über alle Militäroperationen in Afghanistan. Zwischen den Regierungen Afghanistans und der USA blieb das ganze Jahr über die Vereinbarung über ein Bilateral Security Agreement umstritten und wurde bis Jahresende auch nicht unterzeichnet. Dieses soll Rolle und Status der ab 2014 in Afghanistan verbleibenden US-Truppen regeln. Hinsichtlich der Auseinandersetzungen mit den Taliban kam es das ganze Jahr hindurch zu Kampfhandlungen mit und Anschlägen durch die Rebellen.

Auch der Irak blieb 2013 von Gewalt geprägt. Ziel von Anschlägen der irakischen Al-Qaida wurden sowohl Angehörige und Einrichtungen des schiitischen Bevölkerungsteils als auch der Sicherheitskräfte. Nach UN-Angaben wurden allein 2013 über 7.800 Zivilisten getötet. Zuletzt hatte es 2008 mehr Todesopfer gegeben. Darüber hinaus konnte auch die politische Krise im Irak nicht gelöst werden.

Im Jemen dauerten die Kämpfe gegen Al-Qaida in the Arabian Peninsula (AQAP) 2013 unvermindert an. Allein bei einem Angriff am 6. Dezember auf den Komplex des Verteidigungsmi- 


\section{AKUF-Kriegsdefinition}

,Krieg' definiert die AKUF in Anlehnung an den ungarischen Friedensforscher István Kende (1917-1988) als einen „gewaltsamen Massenkonflikt, der alle folgenden Merkmale ausweist:

a) an den Kämpfen sind zwei oder mehr bewaffnete Streitkräfte beteiligt, bei denen es sich mindestens auf einer Seite um reguläre Streitkräfte (Militär, paramilitärische Verbände, Polizeieinheiten) der Regierung handelt;

b) auf beiden Seiten muss ein Mindestmaß an zentralgelenkter Organisation der Kriegführenden und des Kampfes gegeben sein, selbst wenn dies nicht mehr bedeutet als organisierte bewaffnete Verteidigung oder planmäßige Überfälle (Guerillaoperationen, Partisanenkrieg usw.);

c) die bewaffneten Operationen ereignen sich mit einer gewissen Kontinuität und nicht nur als gelegentliche, spontane Zusammenstöße, d.h. beide Seiten operieren nach einer planmäßigen Strategie, gleichgültig ob die Kämpfe auf dem Gebiet eines oder mehrerer Gesellschaften stattfinden und wie lange sie dauern."

Kriege gelten als beendet, soweit Kampfhandlungen dauerhaft, d.h. für mindestens ein Jahr, eingestellt bzw. nur unterhalb der AKUF-Kriegsdefinition fortgesetzt werden.

Bei einem ,bewaffneten Konflikt' handelt es sich um gewaltsame Auseinandersetzungen, bei denen die Kriterien der Kriegsdefinition nicht in vollem Umfang gegeben sind.

nisteriums in der Hauptstadt Sanaa starben über 50 Menschen und mehr als 200 wurden verletzt. Auch die USA blieben in diesem Krieg mit Drohnenangriffen beteiligt.

Die Gewalt in den nordkaukasischen Republiken der Russischen Föderation hielt auch 2013 an. Hier kämpften islamistische Rebellen für ein Kaukasisches Emirat. Neben Dagestan, Inguschetien und Tschetschenien war auch Kabardino-Balkarien erneut von dem Konflikt betroffen. Das meiste Aufsehen erregten 2013 Operationen der Rebellen außerhalb des eigentlichen Konfliktgebiets: Am 21. Oktober sowie am 29. und 30. Dezember verübten sie Anschläge auf den Nahverkehr in Wolgograd. Dabei starben insgesamt fast 40 Menschen. Dadurch wurden erneut Spekulationen über die Sicherheit bei den Olympischen Winterspielen in Sotschi im Februar 2014 laut.

\subsection{Kriege in Lateinamerika}

In Lateinamerika war 2013 - wie auch in den Jahren zuvor noch ein Krieg zu verzeichnen. Bei diesem handelte es sich um einen innerstaatlichen Antiregimekrieg.

Obwohl in Kolumbien das ganze Jahr über Verhandlungen geführt wurden, dauerte der Krieg zwischen der Regierung und den Fuerzas Armadas Revolucionarias de Colombia (FARC) an. Zunächst beendeten die FARC im Januar einen zweimonatigen Waffenstillstand, der während seines Bestehens zu einem Rückgang der Kampfhandlungen geführt hatte. Der bislang einzige Durchbruch bei den Verhandlungen wurde Ende Mai erzielt, als sich Rebellen und Regierung auf ein Abkommen über ländliche Entwicklung einigten und damit den ersten Punkt auf der Verhandlungsagenda abschließen konnten. Schwieriger und noch nicht abgeschlossen sind die Gespräche zum zweiten Punkt - der politischen Repräsentation. Insbesondere ab Oktober intensivierten sich die kriegerischen Auseinandersetzungen parallel zum Verhandlungsprozess.

\section{Bewaffnete Konflikte}

Seit 1993 beobachtet die AKUF neben Kriegen, die weiterhin den Kern der Datenbank ausmachen, auch bewaffnete Konflikte. Im Jahr 2013 wurden davon acht verzeichnet. Bei vieren handelt es sich um frühere Kriege, bei denen die Kampfhandlungen aber nicht mehr kontinuierlich fortgesetzt wurden, sodass sie nach der AKUF-Definition nicht mehr die Kriterien für einen Krieg erfüllten. Bei drei weiteren Konflikten ist ebenfalls keine Kontinuität gegeben. Diesen ist aber kein Krieg vorausgegangen bzw. in einem Fall liegt dieser bereits längere Zeit zurück. Der verbleibende bewaffnete Konflikt ist durch Kampfhandlungen zwischen nichtstaatlichen bewaffneten Gruppen gekennzeichnet.

Insgesamt sechs bewaffnete Konflikte wurden 2012 beendet und sind daher für 2013 nicht mehr aufgeführt. Drei davon waren Kriege, die noch einige Jahre als bewaffnete Konflikte fortgeführt wurden: Dabei handelt es sich um den Casamance-Konflikt im Senegal, den Kurden-Konflikt im Iran und den Konflikt im indischen Unionsstaat Manipur. Drei weitere Konflikte waren jeweils nur von kurzer Dauer. Diese fanden in Tadschikistan und Burundi sowie zwischen Sudan und Südsudan um die Provinz Abyei statt.

\section{AKUF-Kriegstypen}

Die AKUF unterscheidet fünf Kriegstypen:

A = Antiregime-Kriege, in denen um den Sturz der Regierenden oder um die Veränderung oder den Erhalt des politischen Systems oder gar der Gesellschaftsordnung gekämpft wird.

$\mathrm{B}=$ Autonomie- und Sezessionskriege, in denen um größere regionale Autonomie innerhalb des Staatsverbandes oder Sezession vom Staatsverband gekämpft wird.

$\mathrm{C}=$ Zwischenstaatliche Kriege.

$\mathrm{D}=$ Dekolonisationskriege, in denen um die Befreiung von Kolonialherrschaft gekämpft wird.

$\mathrm{E}=$ Sonstige Kriege.

Zusätzlich unterscheidet die AKUF, inwieweit eine dritte Macht direkt und unmittelbar an den Kämpfen teilnimmt. Bloße Waffenlieferungen, finanzielle Hilfen, Militärberatung, logistische Unterstützung und dergleichen werden jedoch nicht als Fremdbeteiligung gewertet:

$1=$ Es handelt sich um einen Krieg mit Fremdbeteiligung.

2 = Es handelt sich um einen Krieg ohne Fremdbeteiligung. 
Den sechs beendeten bewaffneten Konflikten standen drei neu eskalierte gegenüber: Während die Aufmerksamkeit in Ägypten sich 2013 vor allem auf den Konflikt um den Sturz des gewählten Präsidenten Mursi richtete, fanden auf der SinaiHalbinsel mehrfach Kampfhandlungen zwischen Islamisten und der Armee statt. Bereits 2012 hatte es dort Anfang August einen Anschlag auf einen Grenzposten zu Israel gegeben, bei der anschließenden Militäraktion kamen über 40 Menschen ums Leben. 2013 nun eskalierte dieser Konflikt schrittweise zu häufigeren Auseinandersetzungen.

Auch in Tunesien eskalierte im Schatten des Machtkampfes zwischen Regierung und Opposition ein bewaffneter Konflikt. Im Grenzgebiet zu Algerien - vor allem in der Gegend um den Berg Chaambi - kam es wiederholt zu Kämpfen zwischen Islamisten der Ansar al-Sharia und Sicherheitskräften.

Der dritte neue bewaffnete Konflikt eskalierte 2013 in Mosambik. Dort kündigte die Resisténcia Nacional Moçambiquana (RENAMO), die 1975-1992 in einem lang andauernden Krieg gegen die Regierung gekämpft hatte, an, erneut zu den Waffen greifen zu wollen. Sie beendete damit vorerst ihre Rolle als rein politische Oppositionspartei. Zu bewaffneten Auseinandersetzungen kam es jedoch bislang nur sporadisch.

Zwei der bewaffneten Konflikte im Jahr 2013 wurden im Jahr zuvor noch als Kriege eingestuft. Dabei handelt es sich um den Konflikt zwischen der Türkei und der kurdischen PKK sowie um den zwischen Äthiopien und der ONLF in der OgadenRegion (s.o.).

Ebenfalls früher als Krieg eingestuft war der Konflikt in Algerien. Die Operationen der südlichen Einheiten der islamistischen Rebellen der Al-Qaïda au Maghreb Islamique (AQMI) in Mauretanien, Mali und Niger wurden bis 2011 noch dem Konflikt in Algerien zugerechnet. 2012 wurde dieser Teil der AQMI allerdings einer der wesentlichen Akteure des Krieges in Mali. Die Kämpfe zwischen dem nördlichen Teil der AQMI und Sicherheitskräften im Norden Algeriens wurden seither wegen ihrer geringeren Kontinuität nur noch als bewaffneter Konflikt eingestuft.

Auch dem bewaffneten Konflikt im indischen Unionsstaat Assam war ein Krieg vorausgegangen. Dieser hat sich seit 2011 abgeschwächt. Mit dem Rückgang der Kampfhandlungen ging auch eine Veränderung der Akteurskonstellation einher. Bis 2010 war eine nicht gesprächsbereite Fraktion der United Liberation Front of Assam (ULFA), die für die Unabhängigkeit Assams eintrat, die bedeutendste Rebellengruppe. Seither finden Zusammenstöße mit Sicherheitskräften darüber hinaus vor allem auch noch mit zwei Rebellengruppen der Bodos und der Karbi statt, die ethnische Minderheiten in Assam bilden.

Im Jahr 2011 hatte der Krieg in Libyen auch aufgrund der NATOgeführten Luftangriffe eine große öffentliche Aufmerksamkeit erfahren. Der Krieg konnte wenige Tage nach der Tötung des bisherigen Machthabers Muammar al-Ghaddafi am 20. Oktober nach etwa acht Monaten Dauer als beendet angesehen werden. Trotzdem hielt die Gewalt in Libyen seitdem an. Milizen der siegreichen Opposition kämpften in verschiedenen Teilen des Landes gegeneinander.

\section{Weitere Gewaltkonflikte}

Auch 2013 war eine ganze Reihe von gewaltsam ausgetragenen Konflikten zu beobachten, die nicht unter die AKUFDefinitionen von Krieg und bewaffnetem Konflikt fallen. Die folgenden Beispiele sind rein exemplarisch zu verstehen und erheben insbesondere keinen Anspruch auf Vollständigkeit.

$\mathrm{Zu}$ diesen Konflikten gehörte vor allem - wie bereits in den vergangenen Jahren - die Gewalt zwischen den verschiedenen Drogenbanden in Mexiko. Diese Konflikte forderten 2013 erneut mehr Todesopfer als die meisten Kriege. Auch in anderen Staaten Zentralamerikas ist bereits seit Jahren ein hohes Gewaltniveau zwischen rivalisierenden Banden zu verzeichnen.

Wechselseitige Übergriffe von Milizen verschiedener Bevölkerungsgruppen waren in mehreren Ländern zu beobachten. In Nigeria zeichneten hierfür muslimische und christliche Milizen verantwortlich; in Myanmar waren es buddhistische und muslimische und im Libanon sunnitisch-muslimische und alawitische Milizen. In Kenia verlief die Konfliktlinie nicht zwischen Religionsgruppen, sondern zwischen Viehzüchtern und Ackerbauern. In allen diesen Fällen gab es mehrere Dutzend bis zu über 100 Todesopfern und die Übergriffe erstreckten sich jeweils über mehrere Monate.

Ebenfalls Dutzende Todesopfer forderten Zusammenstöße zwischen Anhängern von Regierung und Opposition sowie Demonstranten und Sicherheitskräften in Bangladesch. Ähnliches gilt für Ägypten, wo nach dem Sturz von Präsident Mursi durch das Militär am 3. Juli mehrere Hundert Anhänger Mursis und der Muslimbruderschaft von Sicherheitskräften bei Demonstrationen getötet wurden.

\section{Konfliktübergreifende Informationsquellen im Internet:}

Al-Dschasira: http://english.aljazeera.net

AlertNet der Thomson Reuters Foundation: http://www.trust. org/alertnet

AllAfrica.com: http://allafrica.com

Amnesty International: http://www.amnesty.org

British Broadcasting Corporation (BBC): http://news.bbc.co.uk Cable News Network (CNN): http://www.cnn.com

Deutsche Welle: http://www.deutsche-welle.de

Human Rights Watch (HRW): http://www.hrw.org

Institute for War and Peace Reporting (IWPR): http://www.iwpr.net

Integrated Regional Information Network (IRIN) des UN Office for the Coordination of Humanitarian Affairs: http://www.irinnews.org

International Crisis Group (ICG): http://www.icg.org

Radio France Internationale: www.rfi.fr

South Asia Terrorism Portal (SATP): http://www.satp.org

UN Department for Peacekeeping Operations: http://www. un.org/Depts/dpko/dpko 


\section{Datenbanken zu Kriegen und bewaffneten \\ Konflikten:}

\author{
Arbeitsgemeinschaft Kriegsursachenforschung (AKUF) der Uni- \\ versität Hamburg (seit 1945): http://www.akuf.de \\ Correlates of War (CoW) (1816-2007): http://www.correlatesofwar. \\ org
}

Escola de Cultura de Pao der Universität Barcelona (seit 2001): http://escolapau.uab.cat

Heidelberger Institut für Internationale Konfliktforschung e.V. (HIIK) (seit 1945): http://www.hiik.de

Uppsala Conflict Data Program (UCDP) der Universität Uppsala (seit 1945): http://www.pcr.uu.se

\title{
Für eine Stärkung des Parlamentsbeteiligungsgesetzes
}

\author{
Kommission „Europäische Sicherheit und Zukunft der Bundeswehr" am IFSH*
}

$\mathrm{D}$ as Grundgesetz verpflichtet die Bundesregierung, wie es im Urteil des Bundesverfassungsgerichts vom 12. Juli 1994 heißt, „für einen Einsatz bewaffneter Streitkräfte die grundsätzlich vorherige konstitutive Zustimmung des Deutschen Bundestages einzuholen." Das Urteil des Bundesverfassungsgerichts zum Lissabon-Vertrag vom 30. Juni 2009 stellt klar, dass der Parlamentsvorbehalt integrationsfest ist und zum unantastbaren Kern der grundgesetzlichen Verfassungsidentität gehört. Seit 2005 bestimmt das Parlamentsbeteiligungsgesetz (ParlBG) die Regeln und das Verfahren der konstitutiven Zustimmung des Bundestags zu bewaffneten Auslandseinsätzen.

Dem ehemaligen Verteidigungsminister Thomas de Maizière scheinen die Mitwirkungsrechte schon seit Langem offenbar zu engmaschig gestrickt. Insbesondere befürchtet er Konflikte zwischen der Ausweitung gemeinsamer militärischer Strukturen und Fähigkeiten in NATO wie EU einerseits und den im Gesetz festgeschriebenen Beteiligungsrechten des Parlaments andererseits. Bereits in den Verteidigungspolitischen Richtlinien von 2011 heißt es: „Ob und inwieweit die Zusammenarbeit in Bündnissen und die sich wandelnde Sicherheits- und Bedrohungslage rechtlichen Anpassungsbedarf nach sich ziehen, wird zu analysieren sein." In einem Interview in der ZEIT vom 16. Mai 2012 bekennt er sich ausschließlich zum „Rückholrecht“ bzw. zu einem „Vetorecht" des deutschen Parlaments. In der vom Verteidigungsminister erlassenen „Konzeption der Bundeswehr" vom Juli 2013 heißt es: „Neben der politischen Bedeutung hat die multinationale Zusammenarbeit erhebliche militärische und praktische Auswirkungen. Sie kann Handlungsoptionen erweitern, die Durchhaltefähigkeit eigener Kräfte erhöhen und Kosten reduzieren; umgekehrt ergeben sich aus Bündnisverpflichtungen planerische Vorgaben an die eigenen Fähigkeiten. Deshalb bedarf die multinationale Zusammenarbeit grundsätzlich der kontinuierlichen politischen Abstimmung und kann das Zurückstellen nationaler Vorbehalte erfordern."

\footnotetext{
* Folgende Mitglieder der Kommission haben die Stellungnahme erarbeitet bzw. tragen sie mit: Detlef Bald, Jörg Barandat, Michael Brzoska, Hans-Georg Ehrhart, Hans-Günter Fröhling, Jana Hertwig, Sabine Jaberg, Berthold Meyer, Reinhard Mutz, Winfried Nachtwei, Bernhard Rinke, Jürgen Rose, Claus von Rosen, Paul Schäfer, Michael Staack.
}

Das eröffnet Raum für Spekulationen, aber auch für politische Initiativen. So plädieren Andreas Schockenhoff und Roderich Kiesewetter von der CDU für einen parlamentarischen Vorratsbeschluss - also die Erteilung einer Generalvollmacht für die Regierung für den Einsatz der Bundeswehr -, um auf dieser Basis der Exekutive das „Einsatzrecht“ und der Legislative das „Rückholrecht“ zuzuweisen. Auch die Wissenschaft beteiligt sich mit Vorschlägen zur Ergänzung des ParlBG. Nach einem Vorschlag aus der Stiftung Wissenschaft und Politik sollte die Zustimmungspflicht für die Teilnahme deutscher Soldatinnen und Soldaten an Einsätzen zwei Ausnahmen umfassen: Die bisherige Praxis deutscher Mitwirkung an den Hauptquartieren der integrierten Kommandostruktur sollte beibehalten und der Entwicklung zu mehr Flexibilität angepasst werden. Darüber hinaus sollte die Teilnahme an der bündnisgemeinsamen Aufklärungs- und Führungsfähigkeit sichergestellt werden.

Eine andere Diskussionslinie zielt auf eine Schließung von Lücken im ParlBG. Bedarf für eine Stärkung gibt es beispielsweise in der Frage eines Einsatzes von Spezialkräften. Neue technologische Möglichkeiten der Fernwirkung, wie ein Einsatz bewaffneter Drohnen, auch vom Boden der Bundesrepublik aus, oder im Rahmen eines „Cyberwar“, könnten zu Lücken in der Parlamentsbeteiligung führen, die es präventiv zu schließen gilt.

Die jüngeren Diskussionen um das ParlBG berühren aber nicht nur aktuelle Fragen der internationalen Integration der Bundeswehr und andere Grenzfälle der Parlamentshoheit über die Bundeswehr. Im Kern geht es um den Stellenwert, welcher der parlamentarischen Mitwirkung beim Auslandseinsatz generell zukommt.

- Entwickelt sie sich zum Ballast für die deutsche Sicherheitspolitik?

- Torpediert der Bundestag mit seinen Verfahren und Entscheidungsbefugnissen militärische Kooperation im Bündnis oder der EU, zumal diese eingedenk knapper Kassen arbeitsteiliger werden dürfte?

- Soll die Parlamentsbeteiligung beim bewaffneten Auslandseinsatz als das institutionelle Rückgrat einer Kultur der Zurückhaltung gestutzt werden, weil auf Deutschland künftig mehr militärische Verantwortung zukommt? 